\title{
OPINION
}

\section{Randomised controlled trials in general practice: time for international collaboration}

\author{
Martin R Underwood, Yvonne H Carter
}

Unintentional injury to children aged under 5 caused 428300 deaths globally in 1990. Not surprisingly, the majority are in the developing world, with 740 deaths annually per million of the population aged less than 5 , compared with 249 per million a year in developed countries. However, injuries account for $15.6 \%$ of deaths after the perinatal period in this age group in the developed regions, compared with $4.1 \%$ in developing regions. ${ }^{1}$ Thus, although in absolute terms injuries are a problem that needs to be tackled worldwide, proportionally they are a greater problem in the developed world.

Either way, the size of the problem means there is a clear need for initiatives of proven value that can be used to reduce mortality. ${ }^{2}$ The greater prosperity and relative importance of childhood injury in the developed world mean that this is where robust research is most likely to be performed. However, studies that included developing countries could inform a global injury prevention strategy.

Young children, are less competent than adults in assessing and avoiding risk; thus society has an obligation to develop initiatives to reduce their injury rate. Such initiatives can be broadly divided into:

(1) Community based interventions, that affect mainly the environment outside the home, or safety of items brought into the home, such as encouraging (or enforcing), the use of child seats in cars or improving the safety of children's toys.

(2) Educational initiatives targeted at parents to improve safety behaviour such as avoidance of burns and scalds and the correct use of child seats in cars.

Although some public policy initiatives have been tested in a comparative manner either using historical data or similar areas with no intervention as controls, few such initiatives have been evaluated using randomised controlled designs. ${ }^{3}$ Subjecting the introduction of public policy initiatives to the rigour of a controlled trial, possibly using a no intervention control district or districts, presents considerable difficulties. ${ }^{4}$ If a small number of districts were used in such studies it would be difficult to generalise from the results or even to be certain that any differences found were due to the intervention. The additional problems involved in ensuring adequate randomisation and an element of blinding mean that it is unlikely that such studies will be performed.
Educational and support programmes, however, can and should be subjected to rigorous scientific evaluation before they are introduced on a large scale. Roberts et al, in a systematic review of eight trials, calculated the pooled odds ratio of the effect of home visiting to prevent unintentional injury to be 0.74 (95\% confidence interval 0.6 to 0.92$).^{5}$ All but one of these studies selected mothers of children perceived to be at high risk of injury and all provided labour intensive interventions. Although the rate of injury among children at high risk means there is, in relative terms, a large potential benefit from such interventions, most children are not at high risk.

To achieve a significant absolute reduction in the rate of injury any programme should be universal. It is unlikely that resources will be available to fund individual professional or non-professional support, of the type analysed by Roberts, for all new mothers. A more realistic scenario is the provision of an injury prevention programme aimed at all pregnant women or parents of preschool children.

This could include additional classes, leaflets, or other educational material provided during antenatal care, the postpartum period, or routine child health surveillance. Additional support, or the provision of safety equipment, could also be targeted at families perceived to be at higher risk based perhaps, on home accident risk audits.

Existing studies have failed to demonstrate any convincing injury reductions from educational initiatives. However, an effect on parental behaviour, an interim outcome measure, has been shown. ${ }^{3}$ One review reported such positive effects in five out of seven randomised controlled trials. ${ }^{6}$

Nevertheless, educational and support programmes are an attractive approach to reducing childhood injury, not least because if effective they would be relatively economical. The failure of existing studies to show a reduction in injury rates may reflect inadequate sample size rather than an ineffective intervention.

In future such trials may be well advised to concentrate on those under 5 for the following reasons.

(1) A home education programme will have less impact on older children as they become more autonomous, less under direct parental control and, therefore, more likely to be injured outside the home. e-mail: M.Underwood@ mds.qmw.ac.uk 
(2) Younger children are injured more often which makes it, statistically, easier to demonstrate any benefit.

(3) Parents of younger children will be more accessible for an education and support programme than those of older children.

(4) If a cluster randomisation is used, increasing the number of individuals in each cluster will provide little statistical advantage (see below).

In the developed world one in 4000 of children aged less than 5 will have a fatal accident each year. Accordingly a trial to demonstrate a reduction in mortality as the end point would have to be unrealistically large. It would, however, be possible to test, in a randomised controlled trial, the hypothesis that:

An intensive parental educational and support programme will reduce hospital admissions, due to injury in the first five years of life by one fifth.

UK figures show that $2 \%$ of children aged under 5 will be admitted to hospital with an injury annually; one quarter of these will be moderate or severe. ${ }^{7}$ Thus allowing for repeat admissions, about $9.6 \%$ of children will have been admitted to hospital with an injury at least once by their fifth birthday. To show a reduction in admissions by one fifth to $7.7 \%$ (significant at the $5 \%$ level and with a power of $80 \%$ ), for five years of intervention and data collection, 3463 children in each group would be required.

Randomisation by family would be inappropriate because unintentional exposure of the control group to the intervention could occur if they attended the same health clinics as the intervention group,

This means that randomisation by "health care unit" is preferable (cluster randomisation). Unfortunately, because some of the differences between groups attending different health care facilities will be due to factors other than the intervention, it will be necessary to apply an inflation factor to the sample size calculation. ${ }^{8}$ The inflation factor for the effect of randomising by cluster is given by the formula: $-1+(\tilde{n}-1) \rho(\tilde{n}$ is the average number of individuals in a cluster and $\rho$ is the intracluster correlation coefficient (ICC) a measure of the extent to which the behaviour of individuals in a cluster is affected by their cluster membership). A value of one for the ICC means that all the individuals in the cluster behave identically manner, a value of zero means that their behaviour is unaffected by cluster membership. The cluster effect can be reduced by matching the treatment and control centres for known potential confounding variables, but it cannot be avoided completely.

In the UK $98 \%$ of the population are registered with a general (family) practice. Services are free at the point of use, and provide, either directly or indirectly, most community maternal and child health services. Assuming a low ICC $(\rho)$ of 0.01 , and an average cluster size $(\tilde{\mathrm{n}})$ of 100 children (the number born annually into a typical British general practice) a doubling of the sample size is required. If the true value of $\rho$ is larger the sample needed would increase greatly and vice versa if smaller clusters were used.

This sample size exercise demonstrates that to perform a trial of this nature in the UK at least 70 general practices would need to be involved for five years. A trial of this magnitude is costly and unlikely to be repeated. It should be designed therefore, to be as robust and as generalisable as possible. If such a trial was performed in a single country the results could not necessarily be applied internationally. A collaborative study, involving centres in a number of different countries with different economies, health care systems, and injury risk profiles might, therefore, be preferable. If a mixture of developed and developing regions were included robust conclusions could be drawn for the main trial end points and substudies of national interest could also be performed. However, international differences will affect both the injury rate and the likelihood of a child being admitted to hospital after an injury. These differences will make calculating the sample size more complex and could increase the sample size required.

The education package should be tailored to the different risks in participating countries. For example, drowning in a swimming pool is commoner in southern compared with northern Europe, while the reverse is true for burns from domestic heaters. What would be tested is, the pragmatic hypothesis that an education and support package will reduce injuries, rather than explanatory hypotheses identifying which part or parts of the package has the effect.

Although international cooperation increases the complexity of such a trial, if positive results are found there would be a mandate to institute the programme worldwide. If negative results are found we will know conclusively that the solution to this problem lies elsewhere. The European Union through its BIOMED II programme, provides concerted action funding to allow European collaborators to meet and produce detailed trial protocols to be submitted to funding bodies in individual countries. Similar pump-priming from a supranational agency, such as the World Health Organisation would allow potential collaborators to produce a robust trial design. It is time for international cooperation to design a definitive multicentre trial to decide if an intensive parental educational and support programme will reduce the global burden of childhood injury.

We are grateful for the helpful comments of the anonymous reviewers of an earlier draft of this paper.

1 Murray CJL, Lopez AD. Global and regional cause-of-death patterns in 1990. Bull World Health Organ 1994;72:447-80.

2 Stone D. Research on injury prevention: time for an international agenda. $f$ Epidemiol Community Health 1996;50:127-30

3 Towner E, Dowswell T, Simpson G, et al. Health promotion in childhood and young adolescence for the prevention of uninten4 tional injuries. London: Health Education Authority, 1996. childhood injuries: evaluation of the Statewide Childhood Injury Prevention Program (SCIPP). Am f Public Health Injury Prevention 
5 Roberts I, Kramer MS, Suissa S. Does home visiting prevent childhood injury? A systematic review of randomised controlled trials. BMF 1996:312:29-33.

6 Bass JL, Cristoffel KK, Widome M, et al. Childhood injury prevention counseling in primary care settings: a critical review. Pediatrics 1993;92:544-80. 7 Walsh SSM, Jarvis SN, Towner EML, et al. Annual incidence of unintentio

8 Hsieh FY. Sample size formulae for intervention studies with the cluster as unit of randomisation. Stat Med 1988;8: 1195-201.

\title{
DISSENT
}

\section{Going international: what are the implications?}

\author{
Charles Larson
}

In absolute terms, the magnitude of childhood mortality and disability caused by injury has become a priority public health problem, recognized by developed and, more recently, developing countries worldwide. By the year 2020, injuries will constitute one of the most important, if not the leading, causes of disease burden worldwide. ${ }^{1}$ For this compelling reason alone, there is an urgent need for scientifically based knowledge with which to guide the development of effective injury prevention programs. This implies, at the very least, the presence of three important conditions: (1) the capacity to conduct relevant, well designed research in developing, as well as developed countries, (2) the existence of funding to support such research, and (3) the ability to effectively apply research findings to health policies and program development.

Underwood and Carter's Opinion in this issue points to the need all countries share in identifying "...initiatives of proven value". They argue that this is inhibited, not only by limited scientific support for community-or family based injury prevention programs-but by studies weakened due to inadequate sample size or non-randomized designs. Underwood and Carter do identify important exceptions, such as Robert's meta-analysis of antenatal home visitation and its relation to reduced early childhood injuries. They take the position, however, that such interventions will not significantly impact on the global problem of childhood injury because they are too expensive and high risk, as opposed to universal or population based programs. As a strategy to overcome these limitations, Underwood and Carter recommend the development of collaborative, multinational studies, which mix the experiences of developed with developing countries. They argue that a randomized field trial of a varied (site appropriate) education and support intervention package could conclusively answer whether or not such programs work.

At what point is a randomized field trial the appropriate design of choice? Given the lack of support from observational studies for "stand on their own" universal educational or support injury prevention interventions, the cost and resource consumption required of a randomized field trial is not justified. This would be particularly the case in a developing country. It is important to understand that within developing countries, there also exists the great need to support descriptive and observational studies, upon which they can establish their own knowledge base. This information can then be applied to appropriately formulated injury intervention programs. This implies greatly increased funding, beyond current levels, from developed countries to support research which is not necessarily tied to their own research agendas.

While international collaboration in injury prevention research is a goal I would heartily support, I caution that such initiatives do carry the potential of doing more harm than good. This is especially the case in settings where resources are already well under optimal. Multicenter trials will be beyond the funding capacities of most developing countries, which ultimately leads to questions of who will fund and control such studies. This, by extension, then leads to consideration of a nation's research agenda and their ability to independently define and pursue health research priorities. It is also the case that well funded, internationally supported studies often engage the best, most productive of a developing nation's researchers. The potential consequence of this is to deflect a precious resource away from other, equally compelling problems.

Beyond the issues of research conduct and funding is the question of the interpretation and application of a study's results, in particular a large, multinational trial. The pooling of outcomes from highly variable settings may produce a result that has little connection with reality. This is analogous to the consideration of pooled, or mean, per capita income in developing countries, a value that is representative of almost nobody. It is conceivable that a specific intervention may be highly productive in a few settings, but not in the majority. The pooled result will rest somewhere in between, its interpretation will be difficult, and its applicability highly questionable. In the end, one 
returns to the need to design studies with sufficient power to address the issue of interpopulation variability in response.

There is also the question to whom would one generalize the results of an investigation with an international study population? Will a mean change in injury rates be the appropriate outcome? Is this likely with an educational intervention in light of existing knowledge? Is the focus on preschool age children justified?

Yes, by all means we should encourage Underwood and Carter's underlying thesis of international collaboration in injury prevention research. This should be done in many settings, and in a manner that facilitates the exchange (not just transfer) of appropriate research technologies and experience. We live in a world which, despite its unjustifiable disparities, is more than ever before vulnerable to shared determinants of health, be these economic, environmental, behavioral, or political. We all stand to gain by working together. The problem is that this is far easier said than done!

1 Murray CJL, Lopez AD. Global burden of disease and injury. Vol I. Boston: Harvard University Press, 1996.

\section{Editorial Board Member: brief biography}

\section{DINESH MOHAN}

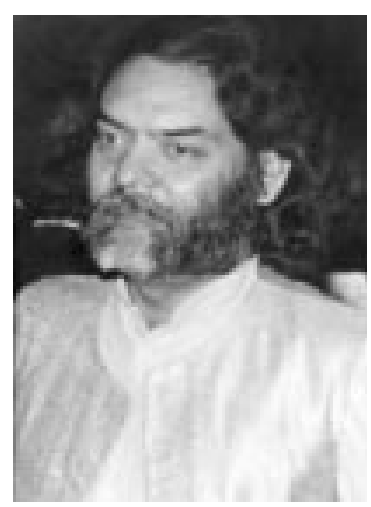

Dinesh Mohan has been involved in injury control research for the past 25 years. $\mathrm{He}$ obtained a bachelor's degree in mechanical engineering from the Indian Institute of Technology, Bombay, a master's degree in mechanical and aerospace engineering from the University of Delaware, USA and a $\mathrm{PhD}$ in bioengineering from the University of Michigan, USA. He started his research career at the Biomechanics Department of the University of Michigan Transportation Research Institute at Ann Arbor where he specialised in biomechanics of impacts and human tolerance research. He continued this work at the Insurance Institute of Highway Safety, Washington DC, where he worked with William Haddon and did projects on injuries due to free falls, baseball impacts to the head, evaluation of the effectiveness of airbags in cars, injuries to children in cars, and the clasping strength of adults in holding children in laps in cars.

Currently he is a Professor in the Centre for Biomedical Engineering at the Indian Institute of Technology, Delhi, India, and Head of the WHO Collaborating Centre for Research and Training in Safety Technology. His current interests include research on transportation injuries with a special focus on vulnerable road users and injuries in rural areas. He serves on many national and international advisory groups including the injury prevention programme of the WHO. He is a Vice President of the International Association for Accidents and Traffic Medicine and a board member of the International Research Council on the Biomechanics of Impact. He is on the editorial boards of the journals Accident Analysis and Prevention and Injury Prevention. He has published over a hundred papers and is the coauthor of the book Injury Control: A Global View. He has received the Association for Advancement of Automotive Medicine 1991 Award of Merit for outstanding research in traffic safety, and the International Association for Accident and Traffic Medicine award and medal for outstanding achievement in the field of traffic medicine. 